\title{
Fiber Optic Broadband Ultrasonic Probe for Virtual Biopsy: Technological Solutions
}

\author{
E. Biagi, ${ }^{1}$ S. Cerbai, ${ }^{1}$ L. Masotti, ${ }^{1}$ L. Belsito, ${ }^{2}$ A. Roncaglia, ${ }^{2}$ G. Masetti, ${ }^{3}$ and N. Speciale ${ }^{3}$ \\ ${ }^{1}$ Electronics and Telecommunications Department, University of Florence, 50139 Florence, Italy \\ ${ }^{2}$ Institute of Microelectronics and Microsystems, National Research Council of Italy, 40129 Bologna, Italy \\ ${ }^{3}$ Electronics, Informatics and Systems Department, University of Bologna, 40135 Bologna, Italy
}

Correspondence should be addressed to E. Biagi, elena.biagi@unifi.it

Received 16 July 2010; Accepted 24 October 2010

Academic Editor: P. Siciliano

Copyright (C) 2010 E. Biagi et al. This is an open access article distributed under the Creative Commons Attribution License, which permits unrestricted use, distribution, and reproduction in any medium, provided the original work is properly cited.

\begin{abstract}
An ultrasonic probe was developed by using, in conjunction, optoacoustic and acousto-optic devices based on fiber optic technology. The intrinsic high frequency and wide bandwidth associated both to the opto-acoustic source and to the acousto-optic receiving element could open a way towards a "virtual biopsy" of biological tissue. A Micro-Opto-Mechanical-System (MOMS) approach is proposed to realize the broadband ultrasonic probe on micromachined silicon frames suited to be mounted on the tip of optical fibers.
\end{abstract}

\section{Introduction}

Much recent progress in clinical applications of echography demands for miniaturized and wide bandwidth ultrasonic probes. Wide band ultrasound leads to improved spatial resolution; in addition, it allows spectral analysis of echo signals, a technique that resulted to be effective in clinical diagnosis [1]. However, the diagnostic power of this technique strongly depends on the available ultrasonic bandwidth for differentiating media, due to their frequencyselective interaction.

Our group proposed the design and realization of an ultrasonic source based on optoacoustic effect in 1996, with a metal layer over the fiber optic tip as absorbing target [2]. In 2001 we improved, of about two orders of magnitude, the optoacoustic conversion by replacing the metal absorbing target with a graphite one $[3,4]$.

Other configurations have been developed based on this principle: some make use of graphite powder dispersed in polymeric materials, others require the use of nanostructured materials with gold dipped in polymer matrices [5]. This solution presents the advantage of further extending the band, but has a complicated implementation and requires the use of pulsed laser optical sources, because it exhibits a fairly narrow range of optical absorption. This implies a very complicated and expensive technology.

The transmitting element, depicted in Figure 1, is constituted by a fiber optic on whose tip an absorbing layer is deposed; Thermo-elastic Ultrasound Generation (TUG) takes place when the laser pulse hits this absorbing thin layer and the induced thermal expansion generates a mechanical shock wave.

Two years later, in 2003, we proposed a complete ultrasonic probe by adding a receiving element, based on fiber optic [6]. Several kinds of ultrasonic reception methods by optical interferometry have been proposed in the literature. Almost all use fiber optics to transmit light radiation, but differ in the method of demodulation. In some, the demodulation is carried out downstream of the optical fiber, through complex interferometers [7].

In the so-called interferometric extrinsic receivers, however, the demodulation of the ultrasonic signal into a change in reflected light intensity is performed directly from a structure realized on the tip of the optical fiber. Among the various proposed demodulating structures, some have low transduction sensitivity [8], while those that employ polymeric materials with acoustic impedance closer to that of water show sensitivity comparable with those of classical 


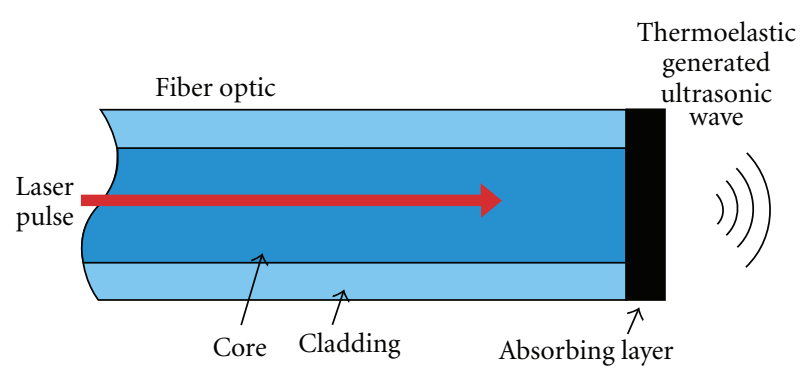

FIGURE 1: Fiber optic ultrasonic source based on optoacoustic conversion.

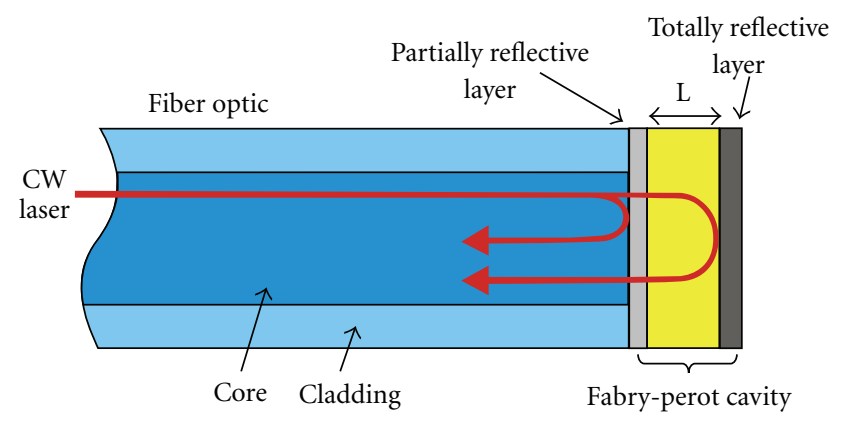

FIGURE 2: Fiber optic ultrasonic receiver based on acousto-optic conversion.

PVDF hydrophones [9]. The advantage of these solutions is, in particular, the large available acoustic bandwidth [10].

The receiving element is constituted by an extrinsic fiber hydrophone, based on a Fabry-Perot interferometer, as depicted in Figure 2. A continuous wave laser beam is launched inside a fiber. The device works as follows: a pressure wave, impinging over the interferometer, modulates its thickness. This change in thickness produces a modification in the interferometer reflectance, defined as the ratio between the reflected and incident light power, and thus induces a change in the light intensity detected by a photodiode.

For the proposed probe, completely based on a fiber optic technology, a strong miniaturization can be obtained, making it suitable for intravascular, endoluminal, and percutaneous applications. Such probes might make it possible to trace a path towards "virtual biopsy"; in fact, these wide band ultrasonic fiber optic probes could be placed in close proximity of a focal lesion by a catheter or a needle [11].

In 2006, the first ultrasonic images obtained with a fully fiber optic ultrasonic probe were presented [5]. In this paper, we present some initial results of the technology development work related to the realization of miniaturized broadband ultrasound sources and detector based on MicroOpto-Mechanical (MOMS) fabrication techniques.

\section{Technological Solutions}

2.1. Objectives. The final objectives of this research are depicted in Figure 3. In both devices, micromachined silicon MOMS (Micro Opto Mechanical Systems) are used to permit the housing of the optical fiber close to the optical layers used

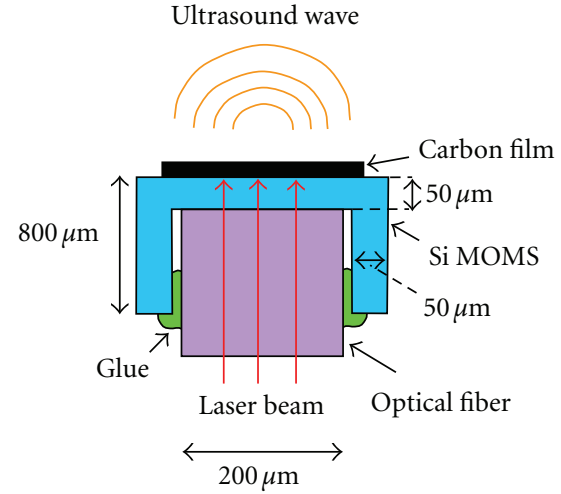

(a)

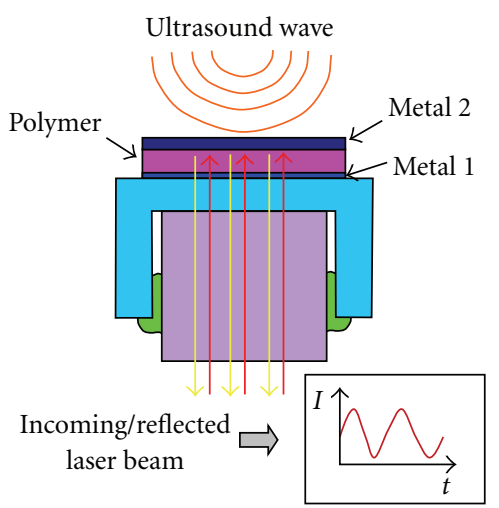

(b)

FIGURE 3: MOMS optoacoustic transmitter (a) and receiver (b) mounted on an optical fiber.
1. Bulk silicon

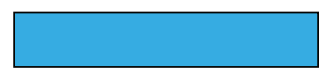

2. Thermal oxidation

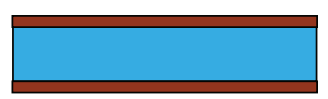

3. Oxide patterning (backside)

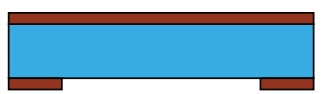

4. Silicon etching (TMAH)

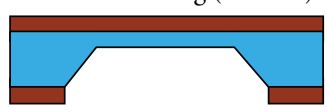

5. Resist spinning

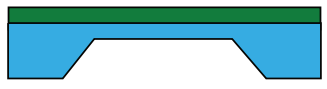

6. Resist carbonization

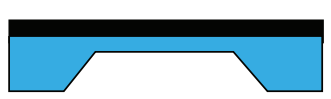

Figure 4: Technological process used to realize carbon layers on micromachined silicon frames.

for ultrasound emission and detection. For the transmitting element, a carbon film with high optical absorption is deposited and patterned on the MOMS upper surface. For the receiver, a planar Fabry-Perot interferometer is realized on a dielectric membrane $\left(\mathrm{SiO}_{2} / \mathrm{Si}_{3} \mathrm{~N}_{4}\right)$ obtained, on the silicon substrate, by backside etching. The interferometer is constituted by a polymer spacer with a low elastic modulus, so that its thickness can be modulated by an 


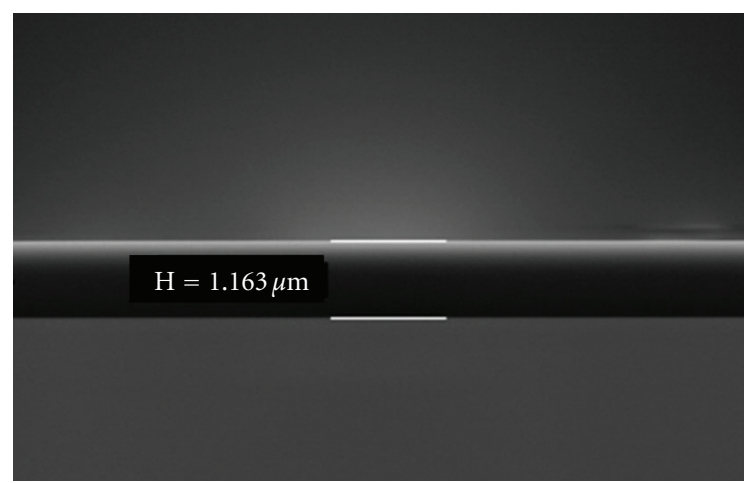

Grafite OIR $\stackrel{1 \mu \mathrm{m}}{\longmapsto}$ EHT $=5 \mathrm{kV}$ Signal A = SE2 Date: 25 Jan 2007 Mag $=32.66 \mathrm{KX} \quad \mathrm{WD}=10 \mathrm{~mm} \quad$ Time: 14:29:05

(a)

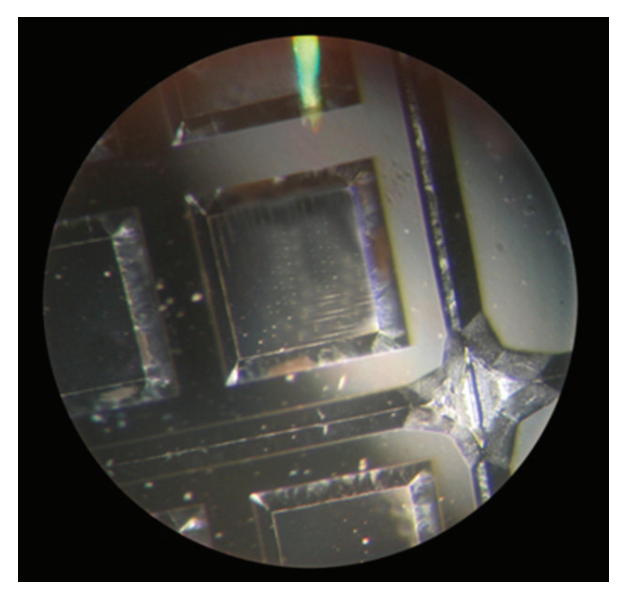

(b)

FIGURE 5: Carbon layer SEM cross-section (a) and optical image, $25 \mathrm{X}$, of the micromachined Si frames (b) obtained in the resist carbonization process.

incoming ultrasound wave. By using the fiber to drive a continuous laser beam on the interferometer, the intensity of the reflected light is modulated by optical interference effects with the same amplitude spectrum of the incoming ultrasound wave.

2.2. Experiments. Some preliminary experiments have been carried out in order to define suitable techniques to obtain layers for efficient optoacoustic emission and acoustooptic detection on planar substrates with photostructurable materials, easy to pattern with lithography.

For the carbon layers, photoresist carbonization on silicon has been investigated as a fabrication technique, using OIR 908-35 positive photoresist from Fujifilm.

The technological process, reported in Figure 4, has been adopted to realize some prototype samples.

Starting from $500 \mu \mathrm{m}$ thick $\langle 100\rangle$ oriented silicon substrates, a thermal oxidation step has been carried out at $1000^{\circ} \mathrm{C}$ in wet environment, yielding a roughly $500 \mathrm{~nm}$ thick silicon oxide layer on both sides of the wafer (step 2). This layer has been used as a mask for the subsequent
1. Bulk fused silica

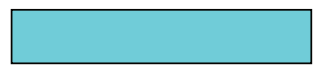

2. Thin $\mathrm{Al}$ deposition $(5,10 \mathrm{~nm})$

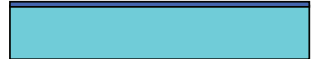

Figure 6: Technological process used to realize planar Fabry-Perot interferometers on fused silica substrates.

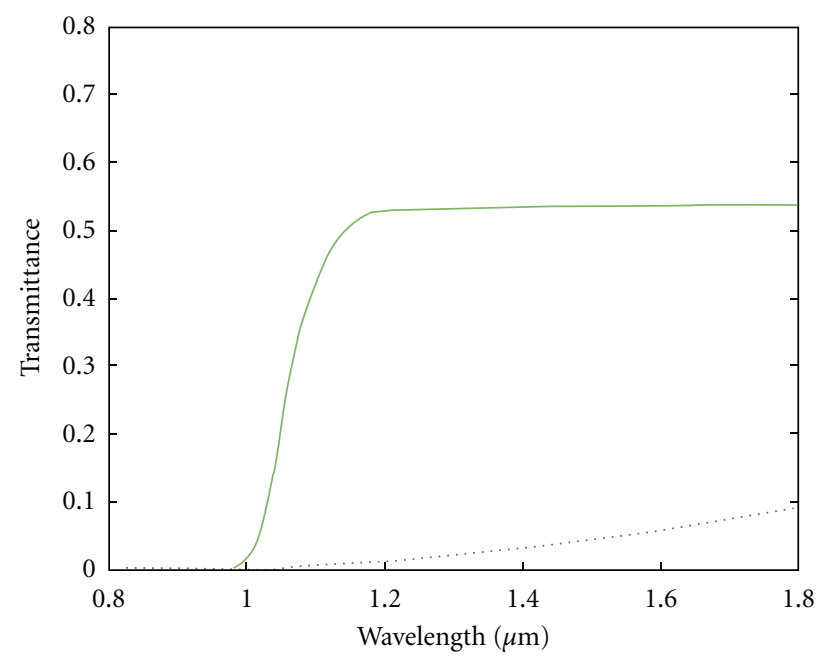

Si wafer + carbon

_ Plain Si wafer

FIGURE 7: NIR transmittance of a carbon layer on a double-side polished silicon wafer compared with the one of a plain silicon wafer.

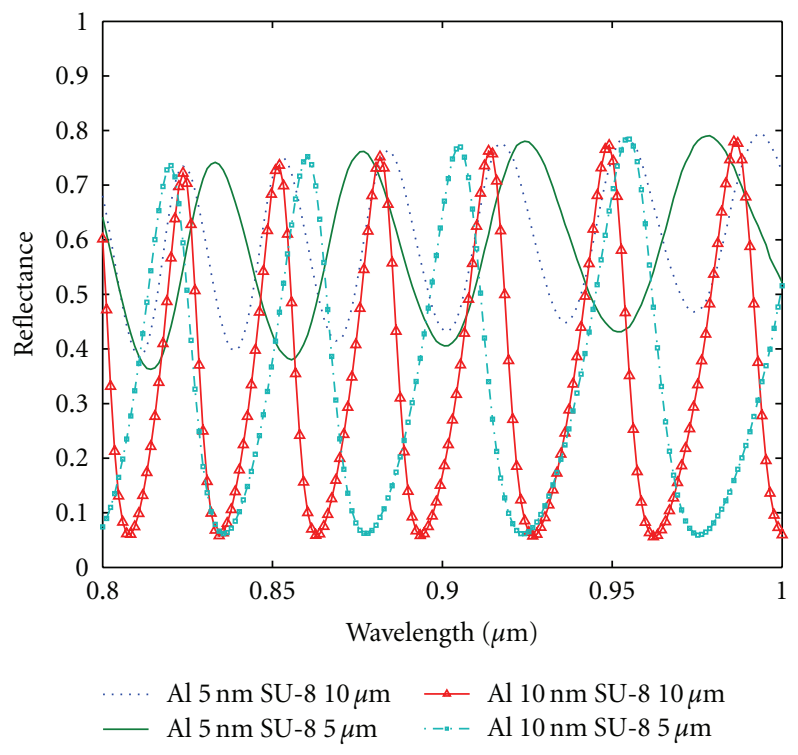

FIGURE 8: NIR reflectance of planar interferometers realized with different thicknesses of the SU-8 spacer and of the thin Al layer. 

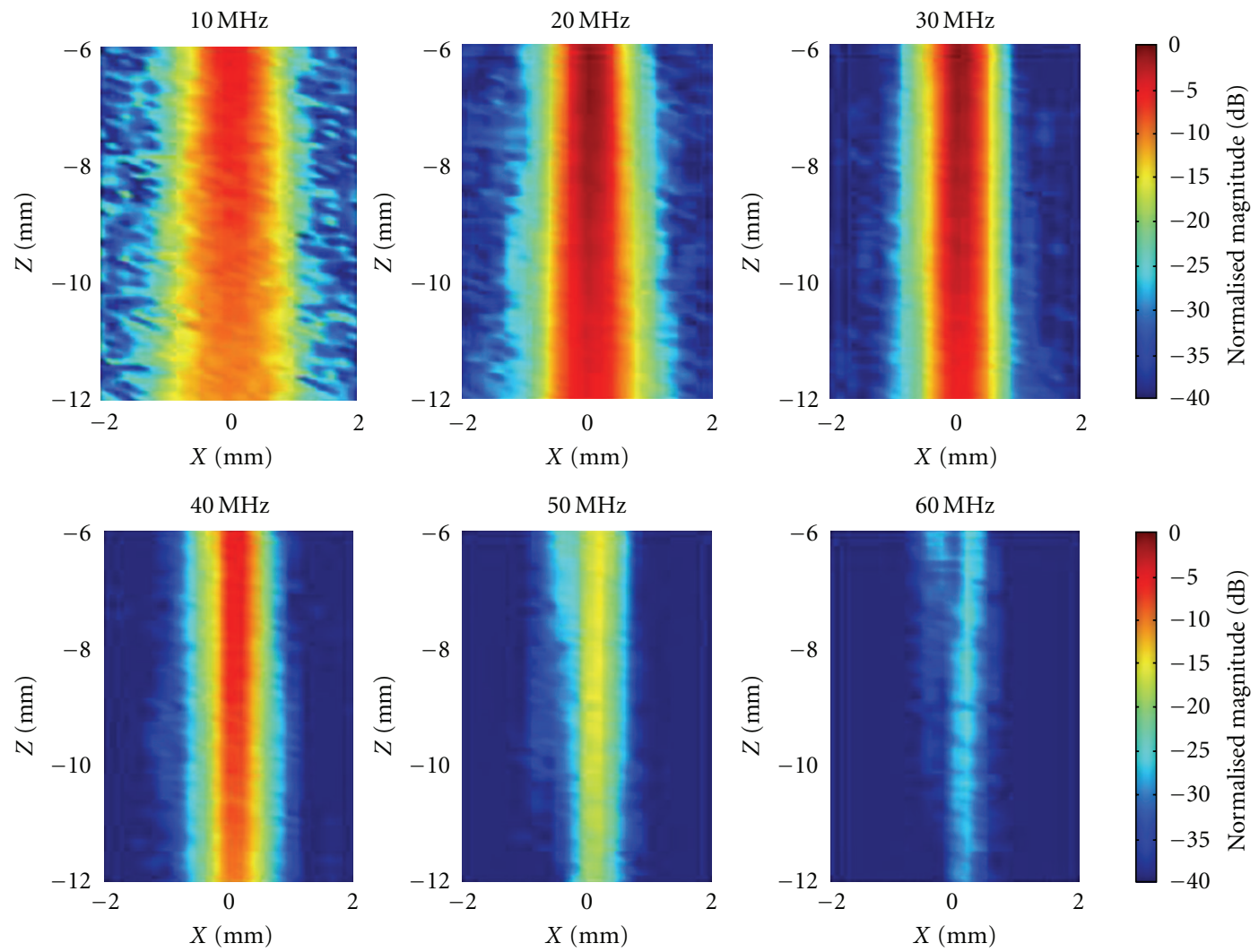

Figure 9: Magnitude of acoustic field longitudinal section, filtered at six different frequencies.

silicon micromachining (step 4), after backside patterning and etching with Buffered Oxide Etch (BOE, step 3). In this way, square silicon membranes with thickness around $200 \mu \mathrm{m}$ and area of $1 \times 1 \mathrm{~mm}^{2}$ have been obtained on the wafer. After removing the $\mathrm{SiO}_{2}$ mask, OIR 908-35 photoresist has been spinned on the wafer front side at $3000 \mathrm{rpm}$, yielding a roughly $4 \mu \mathrm{m}$ thick film. The resist layer has been subsequently carbonized by means of an annealing step performed at $750^{\circ} \mathrm{C}$ in argon atmosphere for 30 minutes. With the same procedure, carbon layers have also been realized on double-side polished wafers for the optical measurements (see Section 3).

A cross-section Scanning Electron Microscope (SEM) image of a carbon layer on silicon obtained with the process described and an optical image of the micromachined silicon frame are reported in Figure 5. It can be observed that the carbon layer obtained after carbonization is roughly $1 \mu \mathrm{m}$ thick, as a result of volume shrinking of the starting resist layer.

For the detector, negative resist SU-8 has been chosen to fabricate the polymer spacer. For the preliminary tests, planar Fabry-Perot interferometers with different thicknesses of the spacer and of the thinnest metal film have been realized on fused silica substrates (pure $\mathrm{SiO}_{2}$ ) according to the process flow of Figure 6. Fused silica has been chosen to mimic the optical properties of the $\mathrm{SiO}_{2} / \mathrm{Si}_{3} \mathrm{~N}_{4}$ membrane that is expected to be adopted in the detectors. Aluminum has been deposited by evaporation to realize both the thin and the thick metal layers of the interferometers. The thickest layer was $50 \mathrm{~nm}$ thick, while two thicknesses have been tested for the thinnest one ( 5 and $10 \mathrm{~nm}$ ). SU- 8 has been deposited by spinning, in $5 \mu \mathrm{m}$ and $10 \mu \mathrm{m}$ thick layers, obtained by varying the spinning speed. The resist layers were exposed to UV light and hard baked at $200^{\circ} \mathrm{C}$ for 5 minutes on a hotplate before depositing the last $\mathrm{Al}$ layer $(50 \mathrm{~nm})$.

\section{Measurements}

The carbon layers on silicon and the Fabry-Perot interferometers on fused silica have been subjected to optical and acoustic characterizations. Results are reported in the next subsections.

3.1. Optical Characterization. The optical transmittance of carbon layers obtained on double-side polished $500 \mu \mathrm{m}$ thick silicon substrates has been measured with a Nicolet 5700 Fourier Transform Infrared (FTIR) spectrometer and compared with the one of a plain silicon wafer (Figure 7).

The measurement shows an evident decrease of the silicon wafer transmittance in the near IR range due to the presence of the heavily absorbing carbon film.

The reflectance of the Fabry-Perot interferometers realized on fused silica has been also measured both in the optical and IR range. In Figure 8, the results of the optical characterizations performed in the NIR range on interferometers with different thickness features are summarized. 


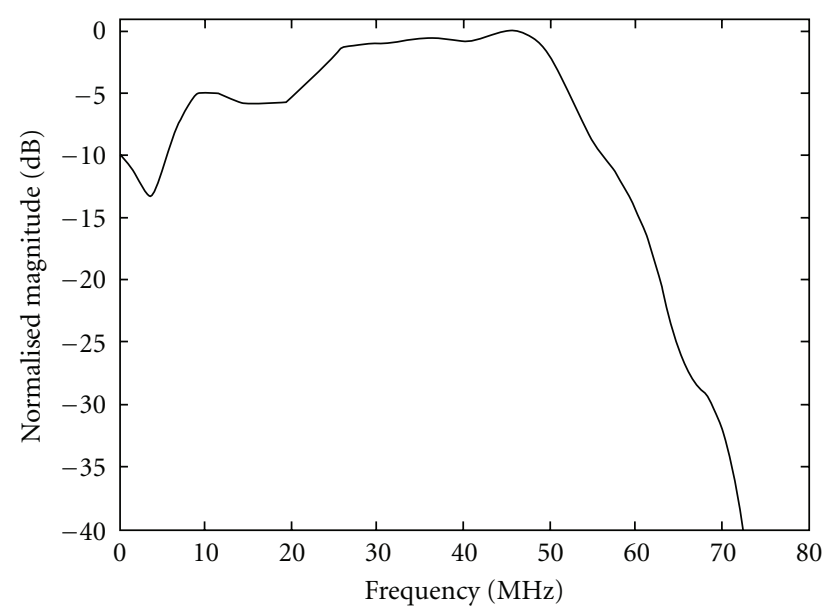

FIGURE 10: Spectral response of the MOMS fiber optic probe.

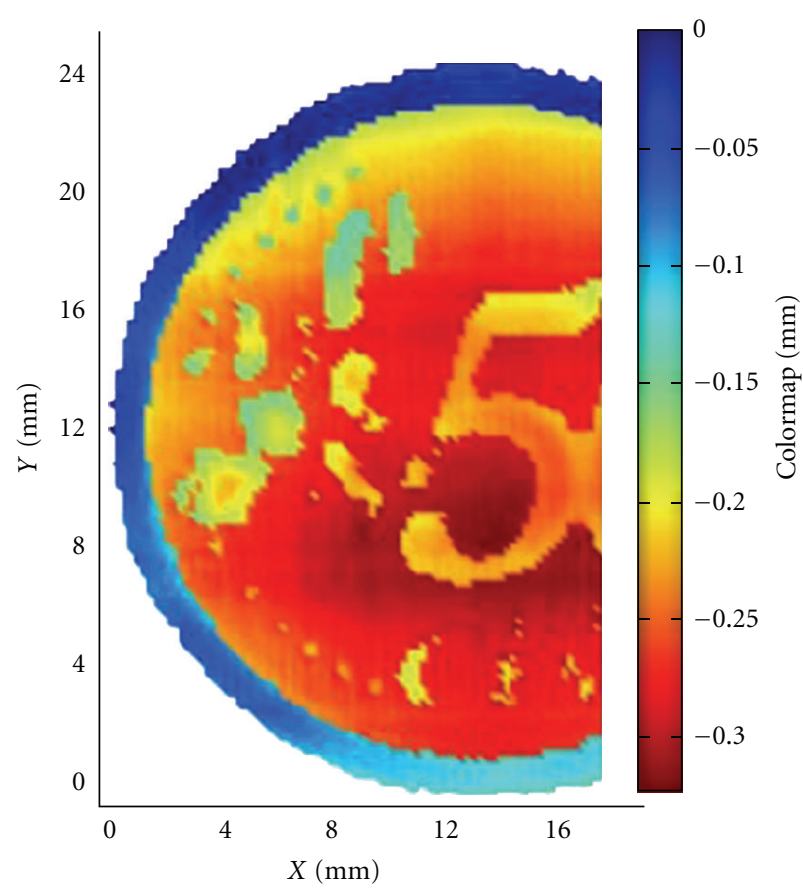

FIGURE 11: Ultrasonic image of a 50-eurocent coin. The coin edge is assumed as a reference $(0 \mathrm{~mm})$.

Evidently, the best optical intensity modulation depth and consequently, the highest optical sensitivity of the interferometer on the spacer thickness is obtained for a SU-8 thickness of $10 \mu \mathrm{m}$ with a $10 \mathrm{~nm}$ thick $\mathrm{Al}$ layer, with an intensity modulation depth of $85 \%$ at $1064 \mathrm{~nm}$ and a maximum slope of $37 \mu \mathrm{m}^{-1}$.

3.2. Acoustic Characterization. A longitudinal section of the acoustic field, generated by the optoacoustic transmitter, was acquired by employing a PVDF membrane hydrophone (Marconi 699/1/00002/200).

In Figure 9, the magnitude of the acoustic field filtered at six different frequencies is reported for 6 to $12 \mathrm{~mm}$ distance from the transducer. The colormap represents the same magnitude level for the all six pictures. The decrease in magnitude observed for high frequencies range is partially due to the hydrophone frequency response (60 MHz cut-off).

The global response of the MOMS fiber optic probe has been obtained by facing the transmitter and receiver elements with each other and acquiring the ultrasonic signal. In Figure 10, the spectral response of the probe is reported.

In Figure 11 a 50-eurocent coin is reconstructed, by elaborating the flight time of the ultrasonic pulse. This image was obtained by using two prototypes of MOMS transmitting and elements.

\section{Conclusion}

The challenge of developing complete transmitting-receiving ultrasonic transducers, based exclusively on fiber optic technology, is extremely important to define a new generation of ultrasonic probes.

The high frequencies and large bandwidth in conjunction with the extreme miniaturization derived from the MOMS technology could open a way towards "virtual biopsy", intended as the possibility for studying and characterizing the nature and health conditions of living tissues "in situ".

Preliminary results are presented on the optoacoustic emission properties of carbon films (thickness around $1 \mu \mathrm{m}$ ), obtained by photoresist carbonization on silicon micromachined substrates, and about the optical and acoustic behaviour of planar Fabry-Perot interferometers composed by thin metal layers and SU-8 negative photoresist on fused silica substrates.

The MOMS technology seems to be suitable to fit the following requirements for the fully fiber optic ultrasonic probes: biocompatibility, repeatable and completely controlled manufacturing process, and low-cost devices production.

\section{References}

[1] E. Biagi, L. Breschi, S. Granchi, and L. Masotti, "Method and device for spectral analysis of an echographic signal," U.S. Patent no. 7509 861, 2009.

[2] E. Biagi, S. Fontani, F. Francini, L. Masotti, and M. Pieraccini, "Photoacoustic generation: all-optical fibre transducers," in Proceedings of the IEEE Ultrasonics Symposium, vol. 2, pp. 921924, November 1996.

[3] D. Menichelli and E. Biagi, "Optoacoustic sources: a practical Green function-based model for thin film laser-ultrasound generation," Journal of Optics A, vol. 3, no. 4, pp. S23-S31, 2001.

[4] E. Biagi, F. Margheri, and D. Menichelli, "Efficient laserultrasound generation by using heavily absorbing films as targets," IEEE Transactions on Ultrasonics, Ferroelectrics, and Frequency Control, vol. 48, no. 6, pp. 1669-1680, 2001.

[5] E. Biagi, A. Acquafresca, S. Cerbai, P. Gambacciani, and L. Masotti, "Fully fiber optic ultrasonic probes for virtual biopsy," in Proceedings of the IEEE Ultrasonics Symposium, pp. 556-559, 2006.

[6] L. Masotti, E. Biagi, F. Margheri, and D. Menichelli, "Optoacoustic generator of ultrasound waves from laser energy supplied via optical fiber," U.S. Patent no. 6519 376, 2003. 
[7] Y. Hou, J.-S. Kim, S. Ashkenazi, M. O’Donnell, and L. J. Guo, "Optical generation of high frequency ultrasound using twodimensional gold nanostructure," Applied Physics Letters, vol. 89, no. 9, 2006.

[8] C. Koch, "Measurement of ultrasonic pressure by heterodyne interferometry with a fiber-tip sensor," Applied Optics, vol. 38, no. 13, pp. 2812-2819, 1999.

[9] C. Koch, "Coated fiber-optic hydrophone for ultrasonic measurement," Ultrasonics, vol. 34, no. 6, pp. 687-689, 1996.

[10] P. C. Beard, A. Hurrell, E. van den Elzen, and T. N. Mills, "Comparison of a miniature, ultrasonic, optical fibre hydrophone with PVDF hydrophone technology," in Proceedings of the International Ultrasonics Symposium, pp. 18811884, October 1998.

[11] A. Acquafresca, E. Biagi, L. Masotti, and D. Menichelli, "Toward virtual biopsy through an all fiber optic ultrasonic miniaturized transducer: a proposal," IEEE Transactions on Ultrasonics, Ferroelectrics, and Frequency Control, vol. 50, no. 10, pp. 1325-1335, 2003. 

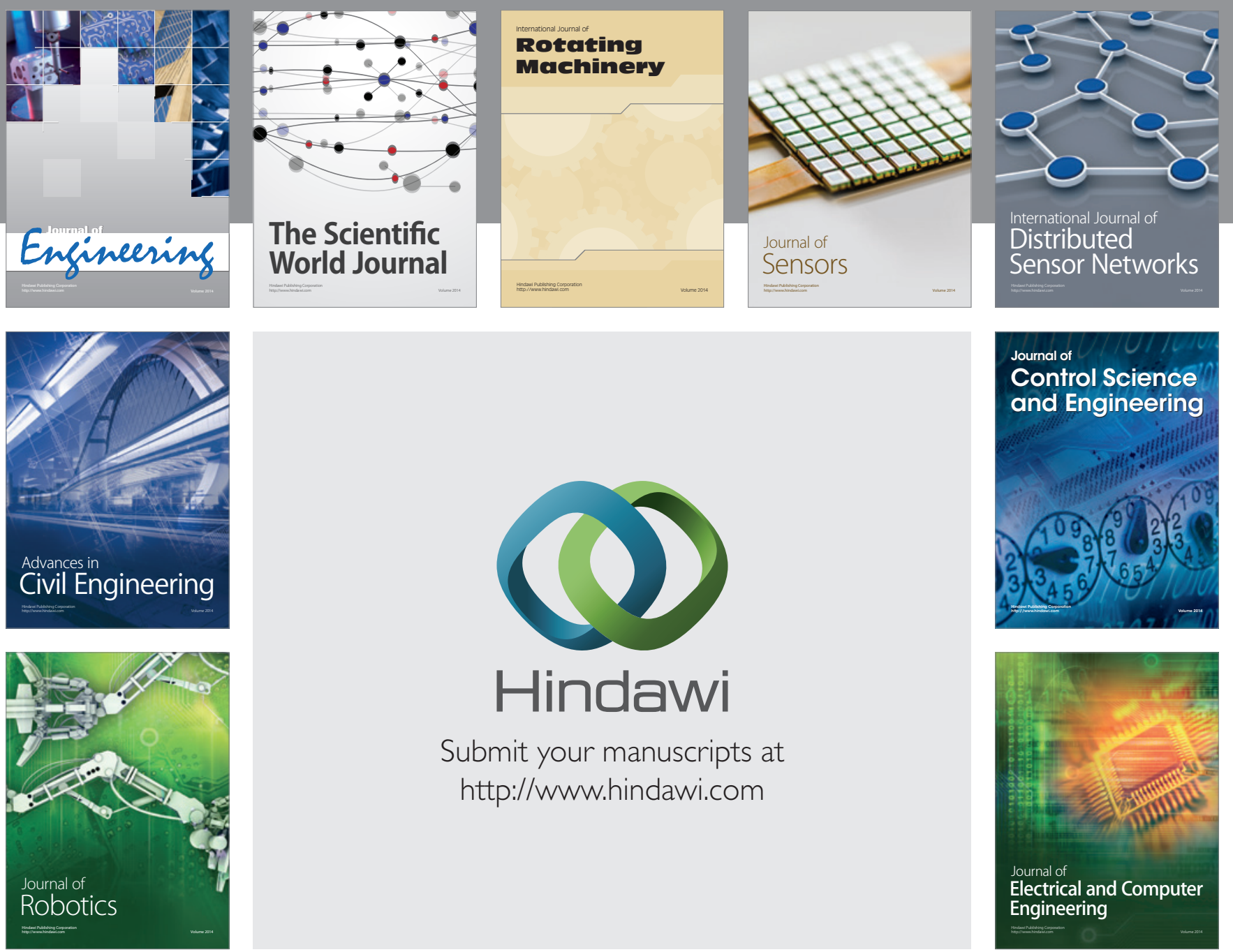

Submit your manuscripts at

http://www.hindawi.com
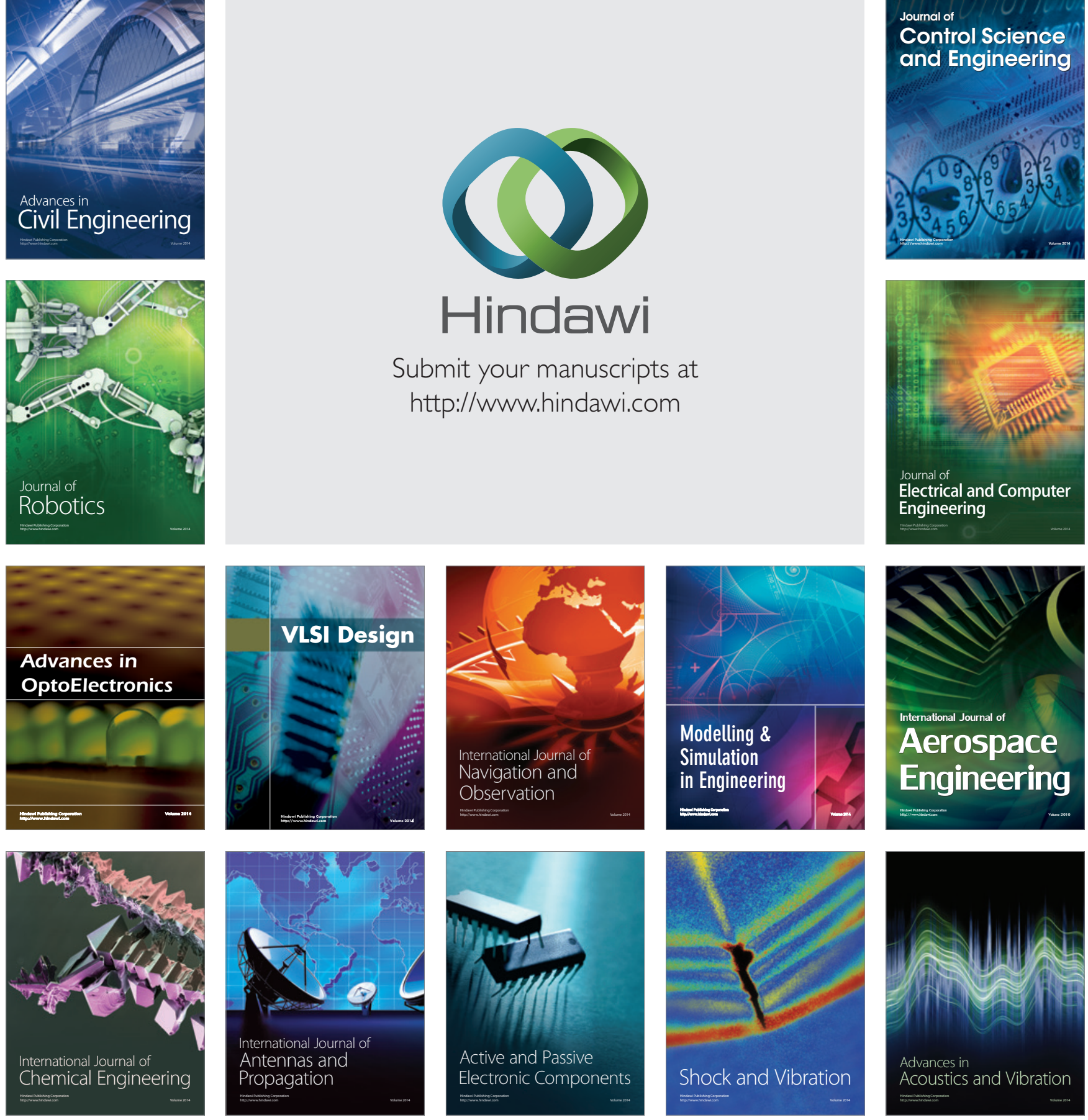